\title{
On the sustainability of global agriculture
}

\section{Editorial}

To explore the impacts on global agricultural systems, as we speak till 2050, is a hectic task. It encompasses essentially the objective to identify the decisions policy makers have to make for the years ahead. They have to decide on the policy to follow to consolidate the objective, that a global population rising to nine billion (or more) can be fed as well as have access to the basic products originating from global sustainable agriculture and forestry (wood, wood pellets, natural fibres, biosynthetic materials, efficient protein production with grasshoppers,...).

The global agricultural system, in the near future (time horizon of about 40years) will undergo an unprecedented set of increasing existing and new anthropogenic and natural impacts. In this system, the demand side is represented by the global population, which will increase from nearly seven billion today, to close to eight billion by 2030 and to over nine billion (or more) by 2050. Some subsets of the global population are and will stay wealthy, creating a demand for a varied, high-quality diet and raw materials from agricultural origin. Additional resources and especially energy and water will be required to enable this type of agricultural production. We can typify this type of agriculture as energy and water resources intensive, hence not durable. This agricultural type will require with an increasing rate, land, water and energy hence it impacts on the three major problem areas of Global as well as Climate Change. The requirement to reduce greenhouse gas emissions and the adaptation of agriculture to a changing climate will be (come) imperative (Figure 1).

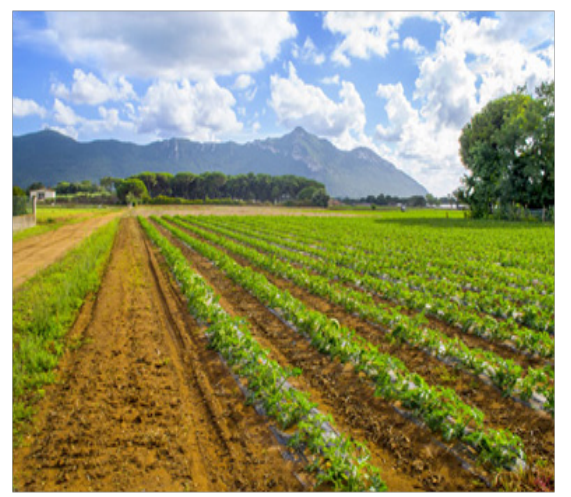

Figure I On the sustainability of Global Agriculture.

Over the time horizon of the next 40years, globalization will continue, exposing agriculture to novel political - economic approaches and impacts. Any one of these approaches will induce substantial impacts on global agricultural systems and hence food security. The sum of the impacts on the water and the energy cycles and climate will become a major factor in a possible decrease of durable global agricultural production. Evidently this will require a structural change and adaptation of global agriculture and forestry to ensure pathways to durable food and raw material production systems. Consolidating its future durable global agricultural production requires the analysis of the impacts cited above in such a way that at least resilience is promoted and obtained. Hence shocks and future uncertainties in global agriculture must be absorbed and anticipated without vital

\author{
Volume 6 Issue 3 - 2017
}

\section{Frank Veroustraete}

Department of Bioscience Engineering, University of Antwerp, Belgium

Correspondence: Frank Veroustraete, Associate Professor in Remote Sensing, University of Antwerp, Department of Bioscience Engineering, Groenenborgerlaan I7I, 2070 Antwerp, Belgium, Email frank.veroustraete@uantwerpen.be

Received: February 03, 2017| Published: February 06, 2017

reductions of global agricultural production rates if major stresses to the food system are to be anticipated and managed. Though recently volatility in food prices for some agricultural products have surfaced the last two years, for the time being, global agriculture still provides large enough quantities of foodstuffs for the majority of the world's population. Yet the global agricultural system fails at two major levels:

i. 925million people experience hunger and worse, a subset of the hungry, are deprived from access to sufficient of the major macronutrients (carbohydrates, fats and proteins). Perhaps another billion suffers from 'hidden hunger': where important micronutrients (such as vitamins and minerals) lack in their diet. This obviously increases the risks of physical and mental invalidity.

ii. Much of the responsibility for three billion people having suboptimal diets originates from global agricultural systems, not being able to produce optimal diets. These optimal diets should be a guarantee for low risks on food related diseases.

iii. In contrast to this first system failure, a billion people are substantially to excessively over-consuming food. And again this induces a public health epidemic involving chronic conditions such as type-2 diabetes and cardiovascular diseases and consequently early death, by diabetes and cardio-vascular complications. One of the impacts of this phenomenon is that a strong impact is felt in the financing of the global health services and organisations.

As mentioned earlier, many agricultural systems on the globe are not sustainable, hence they do not guarantee enough food and raw material production in the future. There are widespread problems with soil loss and degradation even desertification due to erosion, loss of soil fertility, salinisation and other forms of degradation. Rates of water extraction for irrigation are exceeding the rates of replenishment in many places even in some of the richest countries. Over-fishing is a widespread concern worldwide and there is heavy reliance on fossil fuel-derived energy for the synthesis of nitrogen fertilisers and pesticides. 
In addition, emissions to air and water from agricultural production systems are frequently in excess of the levels considered environmentally without impact. Livestock and nitrogen fertilisers are major sources of emissions of the greenhouse gases methane and nitrous oxide, while loss of nitrates and phosphates from soils cause loss of water quality of the rivers flowing through agricultural areas.

Despite the cited environmental impacts of non-durable agriculture and the synergism between actual and future impacts, the global agricultural systems have till recently had relatively little attention from global, national and local policy-makers. Governments over the globe should start developing strategies to obtain durable agriculture and answering the increasing demand by taking a closer look at the cause of food price spikes by establishing a High-Level Task Force on the development Durable Global Agricultural Systems.

The international community, led by the G8 (an inter-governmental political forum of the world's major highly industrialized economies in countries that view themselves as democracies), committed an additional \$22billion over three years from July 2009 towards sustainable agricultural development. While these developments are welcome, it is clear that this type of financial impulse measured on the global scale will not solve the failures of the system. They are a drop on a hot plate, with an ever increasing demand.

A very important issue is the observation that there is the development of a growing intertwining of global agriculture to a broad range of global policy issues. To name a few: "Climate change mitigation and adaptation"; "Durable Energy production, food security and supply"; "Water scarcity and salinisation"; "LUCC, Land Use and Cover Change". On top of this, rural populations without these natural resources lead to an increased urbanization and especially shanty towns and massive population migrations. Sustainability implies the use of resources at rates that do not exceed the capacity of the Earth to replace them for future generations.

Challenges and choices for global sustainability, when postponed in decision making, may be more difficult to implement at a later date than today and be more disruptive. There is a risk of irreversibility of different linked systems, the water cycle and therefore agriculture as well, tipping points in the climate system or high extinction rates for key components of the terrestrial and aquatic biodiversity on which food production systems depend, may inflict major degradations of the systems as we know them now. Five key challenges can be identified in this respect:

i. Balancing future demand for raw materials and food and their supply sustainably;

ii. Addressing the impacts of volatility in the agricultural production systems;

iii. Ending hunger and malnutrition globally;

iv. Implementing the measures required to solve the challenges of a low emissions world;

v. Maintaining biodiversity and ecosystem services while still feeding the world population and still producing enough raw materials (wood, wood pellets, fibers, biosynthetics...).

\section{Conclusion}

Responding to the tightening of food and agricultural raw material supplies is a very complex issue today and a fortiori in the near future, as evidenced by the five key challenges A to E here above. It involves ministries of energy, water resources, transportation, health and family planning, among others to intensively co-operate. Because of the looming impacts of climate change threatening to disrupt global agricultural systems, we even may find that in the near future, energy and water supply policies will have an even stronger impact on future agricultural production than agricultural policies themselves do so far. In short, avoiding a breakdown of global agricultural production chains, will require the mobilization of our entire society. Politicians should better be prepared for this vital challenge today than tomorrow.

\section{Acknowledgements}

None.

\section{Conflict fo interest}

The autor declares no conflict fo interest. 